\title{
Diagnóstico nutricional de pessoas que vivem com HIV/AIDS: revisão de protocolos nacionais e internacionais
}

\author{
Nutritional diagnosis of people living with HIV/AIDS: \\ a review of national and international protocols
}

Erika Aparecida Silveira (https://orcid.org/0000-0002-8839-4520) ${ }^{1}$

Marianne Oliveira Falco (https://orcid.org/0000-0001-9660-7322) ${ }^{1}$

${ }^{1}$ Programa de PósGraduação Ciências da Saúde, Faculdade de Medicina, Universidade Federal de Goiás. Av. Primeira Avenida s/n, Setor Leste Universitário. 74605 050 Goiânia GO Brasil. erikasil@terra.com.br

\begin{abstract}
HIV infection is a predictor of several metabolic and body composition alterations, in addition to malnutrition, anemia, and hypovitaminosis. There are thus specificities of diagnosis and treatment depending on the stage of the disease and the outpatient or hospital environment. The scope of this narrative review was to update the conceptual and theoretical framework regarding the nutritional diagnosis of people living with HIV/AIDS. Current national and international scientific evidence published in scientific articles, recent books, protocols, consensuses, and guidelines were analyzed. The nutritional diagnosis encompasses several clinical aspects and abilities and specific knowledge about HIV/AIDS, such as: clinical history and current clinical situation, food consumption, anthropometric data, body composition, and biochemistry, blood pressure and physical examination. The nutritional diagnosis process was demonstrated in order to provide subsidies for the health teams in the identification of parameters that make it possible to establish a plan for nutritional care to promote, prevent and recuperate the alterations detected in people living with HIV/AIDS.
\end{abstract}

Key words HIV/AIDS, Nutritional assessment, Chronic disease, Malnutrition, Obesity
Resumo A infecção pelo HIV é preditora de diversas alterações metabólicas e de composição corporal, além de quadro de desnutrição, anemia e hipovitaminoses. Existem assim, especificidades de diagnóstico e tratamento conforme o estágio da doença e o ambiente ambulatorial ou hospitalar. O objetivo desse trabalho é apresentar arcabouço teórico e conceitual sobre a realização do diagnóstico nutricional em pessoas que vivem com HIV/ AIDS. Buscou-se evidências científicas atuais nacionais e internacionais publicadas em artigos científicos, livros recentes, protocolos, consensos e diretrizes. O diagnóstico nutricional engloba vários aspectos e habilidades clinicas e conhecimentos específicos sobre essa morbidade. Assim, devido sua complexidade e abrangência, compreende: história clínica e situação clínica atual, consumo alimentar, dados antropométricos, composição corporal, bioquímicos, pressão arterial e exame físico. Foi demonstrado o processo de diagnóstico nutricional de forma a fornecer subsídios para as equipes de saúde na identificação de parâmetros que possibilitem instituir um plano de cuidado nutricional que promova, previna e recupere as alterações detectadas nas pessoas que vivem com HIV/AIDS.

Palavras-chave HIV/AIDS, Avaliação nutricional, Doença crônica, Desnutrição, Obesidade 


\section{Introdução}

Infecção pelo HIV é considerada um importante problema de saúde pública mundial, mesmo com todos os avanços preventivos e terapêuticos obtidos $^{1}$. Um dos principais progressos no tratamento da infecção pelo HIV se refere à terapia antirretroviral (TARV) ${ }^{2}$, bem como ao aumento do acesso a essa terapia em todo o mundo ${ }^{2,3}$. Com relação às pessoas que vivem com $\mathrm{HIV} /$ AIDS (PVHA) existem dois perfis. As PVHA com acesso à TARV, expostas a efeitos adversos, dentre eles, dislipidemias, hipertensão arterial, resistência à insulina, diabetes, alterações na composição corporal e consequentemente aumento do risco de doenças cardiometabólicas ${ }^{4,5}$. E aquelas que não contam com a assistência farmacêutica, que padecem com deficiência imunológica, doenças oportunistas, síndrome de wasting, corroborando para a desnutrição ${ }^{6}$.

Perante a diversidade de doenças, influenciadas ou não por condições socioeconômicas e de insegurança alimentar ${ }^{4-7}$, o tratamento nutricional do HIV/AIDS ganha cada vez mais destaque e relevância ${ }^{6}$, de forma que as equipes de saúde precisam de um arcabouço de conhecimento atualizado, frente às evidências científicas. Esses conhecimentos são importantes para definir terapêutica e conduta clínica, com foco a reduzir agravos, controlar os fatores de risco e melhorar a qualidade de vida das PVHA. São relevantes também para atualizar as equipes de saúde e gestores envolvidas no tratamento da PVHA.

Para instituir qualquer tratamento nutricional é imprescindível realizar diagnóstico nutricional individualizado, criterioso e aprofundado, somente após deve-se definir as condutas e diretrizes do tratamento ${ }^{8}$. Assim, o objetivo desse artigo é apresentar, frente a evidências científicas, critérios para a realizar o diagnóstico nutricional em PVHA.

\section{Metodologia}

Realizou-se uma revisão narrativa da literatura com foco em evidências científicas atuais nacionais e internacionais publicadas em artigos científicos, livros recentes, protocolos, consensos e diretrizes que norteiam os cuidados clínicos e nutricionais na área de HIV/AIDS. As principais organizações e documentos base desse artigo são: Organização Mundial da Saúde (OMS), Ministério da Saúde (MS), The Joint United Nations Programme on HIV/AIDS (UNAIDS), da European
AIDS Clinical Society (EASC), Sociedade Brasileira de Nutrição Parenteral e Enteral (BRASPEN).

Foi também revisada a base de dados US National Library of Medicine National Institutes of Health (PubMed). As palavras chaves utilizadas para a busca foram: body composition, anthropometry, lipodystrophy, hypertension, cardiovascular diseases, syndrome or metabolic, abnormalities, diabetes, dyslipidemias, nutritional diagnosis, antirretroviral therapy, people living with HIV, Acquired Immunodeficiency Syndrome, HIV Infections. Todo o material foi analisado em conjunto pelas autoras e avaliado criticamente com base em conhecimento de epidemiologia, nutrição e experiência prévia em pesquisas aplicadas na área de HIV/AIDS.

\section{Resultados e discussão}

\section{Assistência e Monitorização no acompanhamento em PVHA}

A assistência e monitorização de todas as variáveis que envolvem o diagnóstico nutricional em PVHA deve ser realizada conforme diagnóstico psicossocial, clínico, nutricional e necessidade de acompanhamento do plano de cuidado nutricional instituído. No Quadro 1 está descrita a sistematização do processo de acompanhamento das variáveis, periodicidade das consultas e quando cada aspecto deve ser avaliado.

\section{Diagnóstico nutricional}

A PVHA está susceptível ao acometimento por doenças agudas e crônicas, devido ao mecanismo de ação do HIV, da TARV e das condições sócio ambientais. Desta forma, o diagnóstico nutricional da PVHA, deve caminhar ao lado da assistência farmacêutica e estar um passo à frente do aparecimento da doença ${ }^{9}$. Esta é a principal diferença entre diagnóstico nutricional entre PVHA e aqueles não contaminados pelo HIV, o momento em que deve ser instituído, visto que a PVHA, impreterivelmente sofrerá com as anormalidades metabólicas ou complicações infecciosas. Entretanto os protocolos de assistência clínica às PVHA, dizem respeito principalmente ao tratamento medicamentoso. No que diz respeito às informações sobre nutrição, não há menção de metodologias específicas de aferição nutricional entre PVHA. Há apenas orientações para manejo das complicações advindas do HIV/AIDS ou da $\mathrm{TARV}^{9-13}$. Tal fato corrobora para falha na assis- 


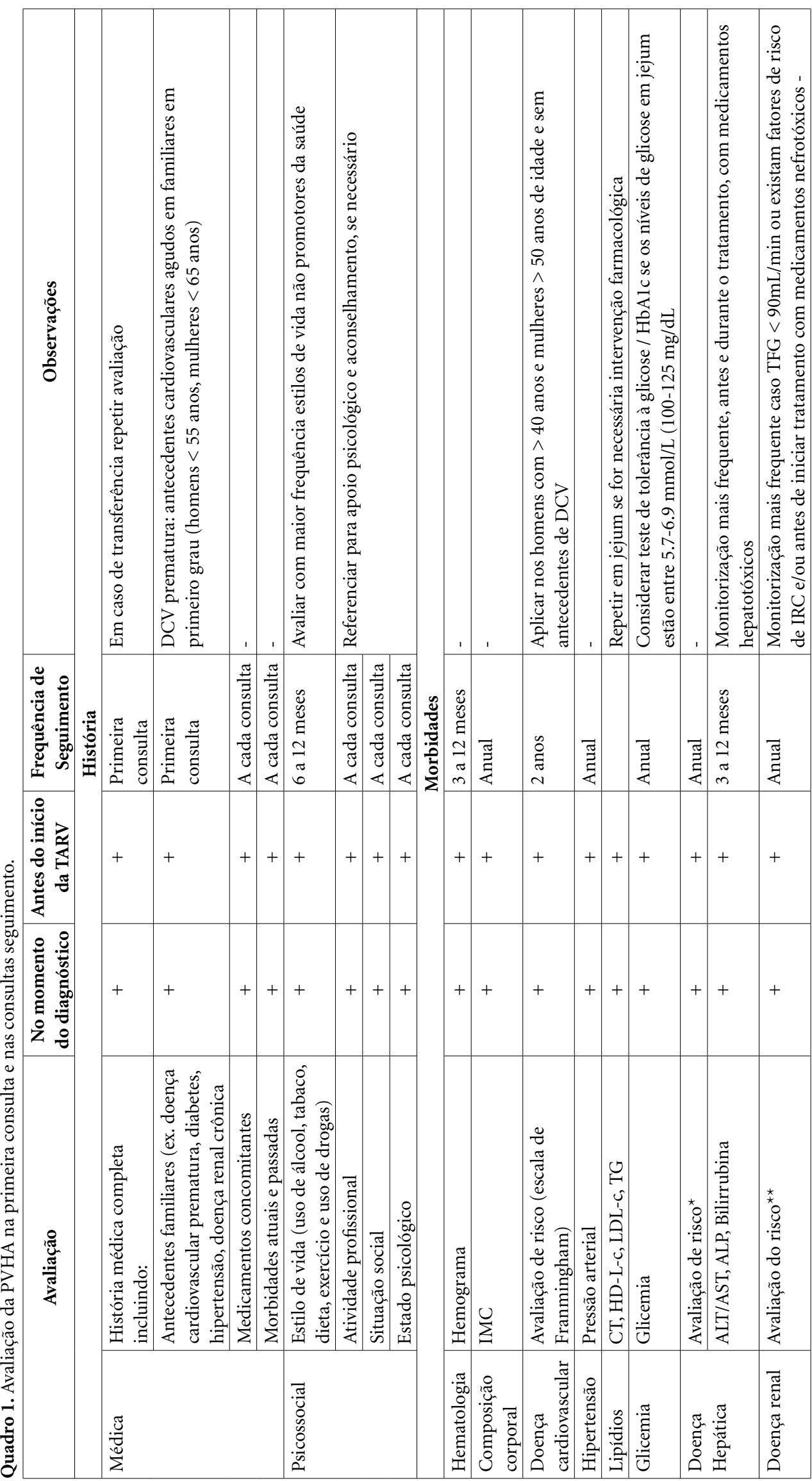


tência integral da PVHA no Sistema Único de Saúde (SUS) e impede que ações de prevenção ao aparecimento de anormalidades metabólicas estejam presentes na assistência primária a esta população.

Com relação aos objetivos do diagnóstico nutricional, considerando todos os aspectos inerentes aos diferentes perfis de PVHA, o profissional deve verificar ${ }^{10-13}$ : desnutrição, perda de peso e presença de síndrome de wasting; deficiência de vitaminas e minerais; perfil de consumo alimentar e hábitos de vida como sedentarismo, tabagismo e consumo de bebida alcoólica; presença de anormalidades metabólicas e fatores de risco cardiometabólicos: dislipidemias, resistência insulínica, intolerância à glicose, diabetes, hipertensão arterial, doença cardiovascular; obesidade geral, obesidade abdominal, adiposidade, lipodistrofia; disfunções de órgãos e tecidos decorrentes do processo inflamatório persistente ou à da toxicidade à TARV: osteopenia, osteoporose, ostemalácia, doença renal e doença hepática ${ }^{6,8-12}$.

\section{Etapas do diagnóstico nutricional}

O diagnóstico é a primeira etapa do cuidado nutricional e envolve um grupo de atividades organizadas que possibilitam o planejamento e monitoramento da intervenção nutricional ${ }^{10,13}$. Assim, para o adequado tratamento e assistência é importante que o diagnóstico seja individualizado e que o profissional reconheça sinais e sintomas que indique necessidade de intervenção nutricional $^{10,13}$.

O diagnóstico nutricional inclui a análise de diversos parâmetros como: história clínica e situação clínica atual, consumo alimentar, dados antropométricos, de composição corporal, e bioquímicos, pressão arterial e exame físico ou semiologia nutricional. Todos esses aspectos são importantes e se complementam não havendo nenhum mais preponderante que o outro e devem ser avaliados inicialmente e monitorados periodicamente, pois a conduta nutricional pode modificar conforme evolução do perfil clínico e nutricional do paciente ${ }^{10,13}$.

\section{Avaliação da história clínica}

Nesta avaliação deve-se verificar o estágio clínico da infecção pelo HIV, pois, após o contato infectante, o processo inflamatório e a ação direta do vírus sobre as células TCD4+, comprometem o sistema imunológico promovendo sintomas e sinais que podem ser identificados na 
avaliação nutricional e ser controlados com um bom cuidado nutricional. Do ponto de vista do comprometimento, são eles ${ }^{10-12,14,15}$ :

- $\quad$ Estágio 1: fase assintomática;

- Estágio 2: perda de peso de origem desconhecida $<10 \%$ em relação ao peso habitual;

- Estágio 3: perda de peso de origem desconhecida $>10 \%$ em relação ao peso habitual, diarreia crônica por mais de um mês, febre persistente inexplicada, candidíase oral, doenças oportunistas (infecções bacterianas graves), alterações hematológicas (anemia, trombocitopenia, neutropenia);

- Estágio 4: síndrome de wasting (consumptiva), encefalopatia e nefropatia associada ao HIV, alto risco de surgimento de doenças oportunistas, sarcoma de Kaposi, linfoma não -Hodgkin e infecção por micobactérias atípicas.

O tempo de diagnóstico de infecção pelo HIV, se em uso ou não da TARV, tempo de uso da TARV, tipo de esquema da TARV, também devem ser investigados, pois são preditores de maior ou menor risco de anormalidades metabólicas, alterações hepáticas, renais e ósseas.

\section{Avaliação do consumo alimentar}

A avaliação do consumo alimentar em PVHA não difere da população em geral. Os instrumentos utilizados, recordatório alimentar de 24 horas, questionários quantitativos ou semiquantitativos de frequência alimentar devem ser capazes de verificar a ingestão de macro e micronutrientes, além de auxiliar na identificação de preferências, intolerâncias e alergias alimentares ${ }^{10,11}$. É necessário que a análise dos resultados desses instrumentos seja quantitativa e qualitativa, pois há macro e micronutrientes cuja ingestão inadequada está associada a alterações bioquímicas e subsequentemente clínicas. A esta maneira deve-se verificar o consumo de alimentos fontes de ferro, cálcio, vitamina C e D, pois a prevenção de anemia e osteopenia devem ser consideradas nesses pacientes ${ }^{13,14}$. Além desses, deve-se investigar o consumo de vitaminas do complexo B, vitaminas A e E, selênio e zinco por estarem relacionados à manutenção do sistema imunológico ${ }^{16-18}$. O consumo de sal, de bebidas alcoólicas e de outros alimentos que constituem risco de dislipidemias, hipertensão arterial, devem ser averiguados. O mesmo para doces em geral, refrigerantes e bebidas açucaradas, principalmente naquelas PVHA com resistência à insulina, diabetes, hipertrigliceridemia, obesidade, lipodistrofia ou doença cardiovascular $^{12,18}$.
Os instrumentos para avaliar consumo alimentar devem ser reaplicados a fim de mensurar alterações de comportamento e consumo alimentar, além de permitir identificar falhas ou sucesso da dietoterapia instituída. Para acompanhar o comportamento alimentar, o recordatório de 24 horas pode ser mais preciso em PVHA, porém não há consenso sobre qual o intervalo para reavaliação ${ }^{10}$.

Em PVHA hospitalizadas alguns sinais e sintomas, tais como, astenia, anorexia, náusea, vômito, disfagia, lesão oral e/ou orofaringeana, pirose febre, diarreia, doenças neurológicas associadas ao HIV e efeitos adversos aos medicamen$\operatorname{tos}^{10}$, entre outros, podem provocar alterações do paladar, dificuldades na deglutição e comprometimento na digestão levando a alteração do comportamento alimentar, o que corrobora para o quadro de desnutrição.

A esta maneira, o monitoramento do consumo alimentar em PVHA institucionalizados deve ser realizado conforme Classificação do Nível de Assistência de Nutrição (NAN), sendo: primário - sem risco nutricional, doença de base que não exija cuidados dietoterápicos, secundário - com risco nutricional, doença de base não necessita de cuidados dietoterápicos ou ainda, aquelas que não apresentam risco nutricional, mas necessitam de cuidados dietoterápico, devido à doenças prévias e terciário - para aquelas com risco nutricional e que precise de dietoterapia ${ }^{8}$. No primário o monitoriamento deve ser de 7 em 7 dias, no secundário a cada 4 dias e no terciário a cada $3 \operatorname{dias}^{8}$.

Deve-se ainda inquerir sobre uso de suplementos alimentares, fitoterápicos e produtos considerados de forma leiga como naturais, pois podem interferir com o tratamento em uso ${ }^{10}$.

Fatores que influenciam as condições de alimentação/nutrição como aspectos da vida pessoal, econômicos, psicossociais também contribuem para um diagnóstico nutricional preciso e devem fazer parte da anamnese nutricional ${ }^{11,19}$.

\section{Antropometria e composição corporal}

A avaliação antropométrica e de composição corporal são importantes e necessárias para o diagnóstico do estado nutricional e presença de múltiplas morbidades como obesidade abdominal, adiposidade, além do acompanhamento da evolução do estado nutricional dos pacientes quer seja ele obeso ou desnutrido ${ }^{10,11}$.

Para avaliar antropometria em PVHA as principais medidas e indicadores são: peso, al- 
tura, índice de massa corporal (IMC), e as circunferências do pescoço, braço, cintura, quadril, coxa, além da relação cintura-quadril ${ }^{9}$. É imprescindível também avaliar a presença de dois agravos que implicam em alterações de composição corporal na PVHA: a síndrome de wasting, que costuma ocorrer em estágios avançados da doença, e lipodistrofia ${ }^{10,11}$.

$\mathrm{Na}$ síndrome de wasting ocorre perda involuntária de peso, geralmente associada à febre documentada por mais de 30 dias ou constante, enfraquecimento crônico, diarreia ( $>2$ evacuações por dia, por mais de 30 dias). Pode haver ainda, perda de massa muscular corpórea (se avaliada, dependendo da disponibilidade do aparelho), maior incidência de infecções oportunistas e tumores (Ex: Sarcoma de Kaposi), multiplicação da carga viral ${ }^{10,13,14,20}$ :

O diagnóstico da síndrome de wasting necessita do peso e IMC, antes da doença como parâmetro de referência, assim os critérios referem-se ao percentual de perda em relação ao tempo. Para facilitar o diagnóstico, pelo menos um dos sintomas abaixo deve estar presente:

- Perda de peso involuntária $\geq 10 \%$ em 12 meses em relação ao peso habitual;

- Perda de peso involuntária $>7,5 \%$ em 6 meses em relação ao peso habitual;

- Perda de 5\% da massa magra corpórea em 6 meses;

- Em homens: Massa magra corpórea < $35 \%$ em relação ao parâmetro habitual e IMC < $27 \mathrm{~kg} / \mathrm{m}^{2}$;

- Em mulheres: Massa magra corpórea < $23 \%$ em relação ao parâmetro habitual e IMC < $27 \mathrm{~kg} / \mathrm{m}^{2}$;

- $\quad \mathrm{IMC}<20 \mathrm{~kg} / \mathrm{m}^{2}$.

Em PVHA as alterações de composição corporal são configuradas como lipodistrofia. A lipodistrofia pode aparecer a qualquer momento, a partir da infecção pelo HIV, independente do uso da TARV ou classe de antirretrovirais ${ }^{21,22}$ e pode se configurar de três formas ${ }^{23,24}: 1$ ) lipohipertrofia, que é o acúmulo de gordura na coluna dorsocervical, nas mamas e abdome; 2) lipoatrofia, caracterizada por redução de tecido adiposo na face e nos membros superiores e inferiores; e 3 ) forma mista. O diagnóstico da lipodistrofia é feito pela concordância de queixas individuais, ou seja, a percepção do próprio paciente, mas também pela percepção da equipe de saúde sobre as alterações de distribuição de gordura corporal, além de avaliação antropométrica e de composição corpora $^{24}$. Antropometricamente a única medida de referência para caracterizar lipodistrofia é a rela- ção cintura-quadril: > 0,95 em homens e entre 0,85 e 0,90 em mulheres $^{19}$. Porém, essa medida antropométrica isolada não trará muita informação se não for avaliada junto à auto percepção do paciente e da equipe de saúde que o acompanha, principalmente em relação a face, abdômen e pescoço ${ }^{25}$.

Para o diagnóstico de obesidade se utiliza os mesmos pontos de corte do IMC para a população em geral ${ }^{12}$. Para obesidade abdominal utiliza-se as medidas da circunferência da cintura aferida entre a última costela e a crista ilíaca e os pontos de corte de $102 \mathrm{~cm}$ para homens e $88 \mathrm{~cm}$ para mulheres ${ }^{26}$.

Para avaliar composição corporal a bioimpedância elétrica tetra polar é um método mais acessível na prática clínica e que apresenta boa confiabilidade de dados. Se for possível, conforme a infraestrutura do local de atendimento da PVHA, realizar avaliação de composição corporal por meio de densitometria corporal total (Dual-energy X-ray Absortiometry - DXA), tomografia computadorizada e ressonância magnética, as quais são técnicas mais acuradas ${ }^{27}$. O DXA pode ser realizado no corpo todo, mas é mais comumente realizado na região de cabeça de fêmur e coluna lombar, sendo utilizado para verificar diversos parâmetros de composição corporal incluindo densidade mineral óssea e a situação de saúde óssea, osteopenia e osteoporose, aspectos a serem investigados e monitorados em PVHA. A tomografia computadorizada e a ressonância, não devem ser utilizadas de rotina, pois implicam emissão de radiação. No entanto, na prática clínica e considerando a realidade de muitos hospitais públicos brasileiros e suas carências em métodos diagnósticos, muitas vezes a única forma de avaliar a composição corporal é através de protocolos de dobras cutâneas ${ }^{24}$. O protocolo de dobras irá por meio de equações preditivas e estabelecidas para a população não contaminada pelo HIV, predizer o percentual de massa magra e de gordura e fornecerá parâmetros para estabelecer comparação entre uma avaliação e outra. Para a PVHA há investigações sobre protocolo de dobras para diagnóstico de lipodistrofia, entretanto, os estudos ainda não são conclusivos ${ }^{28}$.

\section{Avaliação bioquímica}

Avaliação de parâmetros bioquímicos em PVHA é complexa, pois exige conhecimento sobre o metabolismo de macro e micronutrientes, $\mathrm{o}$ impacto da TARV, alterações metabólicas acarretadas pelo próprio vírus, aumento do gasto ener- 
gético, alterações das funções hepática e renal, comprometimento ósseo, presença de doenças oportunistas, principalmente a diarreia, além da existência de situação de segurança alimentar ${ }^{17}$.

Os pontos de corte utilizados para auxiliar no diagnóstico nutricional e planejamento do cuidado dietético, não diferem dos utilizados para a população não contaminada pelo HIV. Os parâmetros mais utilizados são: hemograma (hematócrito, hemoglobina), ureia, creatinina, transaminase glutâmico-oxalacética (TGO), transaminase glutâmico-pirúvica (TGP), proteína total e frações, cálcio, vitamina $\mathrm{D}$, proteína- $\mathrm{C}$ reativa (PCR), vitamina B12, ferro sérico, ácido úrico ${ }^{10,11}$.

Dentre os parâmetros bioquímicos, sabe-se que por muito tempo, a albumina foi utilizada como preditor de estado nutricional, porém com o avanço dos estudos observou-se que isoladamente não é capaz de determinar presença ou ausência de desnutrição. A albumina pode ser utilizada em associação à proteína de fase aguda como preditor independente de morbimortalidade, através do índice PCR/Albumina, parâmetro este validado em 2002, por Corrêa et al. ${ }^{29}$, e é utilizado até aos dias atuais ${ }^{27}$. Ainda pode colaborar na condução e monitorização da terapia nutricional por meio da relação albumina/globulina $^{30}$. No Quadro 2, estão os parâmetros bioquímicos mais utilizados na prática clínica para o diagnóstico nutricional.

$\mathrm{Na}$ fase crônica da doença os marcadores inflamatórios permanecem elevados, o que pode ser investigado em exames de citocinas pró-inflamatórias como TNF-alfa, interleucina 1 e $6, \mathrm{PCR}^{32}$.

A AIDS se caracteriza pela constante replicação viral e redução de linfócitos T CD4 e linfócitos $\mathrm{B}$, afetando progressivamente o sistema imune. Os consensos recomendam início da TARV, tão logo seja realizado o diagnóstico do HIV, independente do estágio clínico e/ou imunológico da PVHA, a fim de reduzir a transmissão da infecção e impedir a redução do CD4 e/ou evolução da carga viral, e assim diminuir a inflamação sistêmica, ${ }^{9}$. O controle da replicação viral também contribui na redução de fatores associados às doenças cardiometabólicas, tais como doença aterosclerótica ${ }^{33}$.

O HIV e a TARV, provocam alterações no metabolismo da glicose e dos lipídeos, assim, também devem ser avaliados e monitorados ${ }^{10}$. Os parâmetros bioquímicos para avaliar a glicose plasmática bem como do perfil lipídico, estão descritos nos Quadros 3 e 4 . Além de verificar alterações de CT, HDL-c, LDL-c, não HDL-c e triglicerídeos (TG) é necessário detectar a existên- cia de dislipidemia. É necessário atentar que para High-density lipoprotein (HDL-c), Low-density lipoprotein (LDL-c) e não HDL-c, os valores foram apresentados apenas como categorias de risco, pois suas referências variam de acordo com a estimativa realizada para o risco cardiovascular ${ }^{34}$.

Os critérios e a classificação das dislipidemias são ${ }^{34}$ :

- Hipercolesterolemia isolada: aumento isolado do LDL-c (LDL-c $\geq 160 \mathrm{mg} / \mathrm{dL}$ );

- Hipertrigliceridemia isolada: aumento isolado dos triglicérides ( $\mathrm{TG} \geq 150 \mathrm{mg} / \mathrm{dL}$ ou $\geq$ $175 \mathrm{mg} / \mathrm{dL}$, se a amostra for obtida sem jejum);

- Hiperlipidemia mista: aumento do LDL -c (LDL-c $\geq 160 \mathrm{mg} / \mathrm{dL}$ ) e dos TG (TG $\geq 150 \mathrm{mg} /$ $\mathrm{dL}$ ou $\geq 175 \mathrm{mg} / \mathrm{dL}$, se a amostra for obtida sem jejum). Se TG $\geq 400 \mathrm{mg} / \mathrm{dL}$, o cálculo do LDL-c pela fórmula de Friedewald é inadequado, devendo-se considerar a hiperlipidemia mista quando o não HDL-c $\geq 190$ mg/dL;

- HDL-c baixo: redução do HDL-c (homens $<40 \mathrm{mg} / \mathrm{dL}$ e mulheres $<50 \mathrm{mg} / \mathrm{dL}$ ) isolada ou em associação ao aumento de LDL-c ou de TG.

\section{Hipertensão Arterial}

Na PVHA a hipertensão arterial pode ter associação com o uso da TARV, mas também com aumento de IMC, da circunferência da cintura, da glicemia de jejum e da dislipidemia ${ }^{36}$. A esta maneira, além dos parâmetros bioquímicos, a pressão arterial, deve ser investigada. São considerados hipertensos os indivíduos com pressão arterial $\geq 140 \times 90 \mathrm{mmHg}^{37}$.

\section{Sinais clínicos de deficiências nutricionais}

Concomitante à avaliação antropométrica, de composição corporal e bioquímica é necessário realizar avaliação de semiologia nutricional. No Quadro 5, é possível observar algumas deficiências nutricionais relacionados à macro $\mathrm{e}$ micronutrientes e suas respectivas manifestações conforme região corporal ou órgão. Assim, é importante conhecer esses sinais e manifestações clínicas de deficiências de proteínas ou de vitaminas e minerais na prática clínica de atendimento desses pacientes (Quadro 5).

\section{Métodos de triagem e avaliação do estado nutricional}

Esses métodos consideram diferentes parâmetros em um único instrumento, sendo o resul- 
Quadro 2. Exames bioquímicos, parâmetros e interpretações em PVHA.

\begin{tabular}{|c|c|c|}
\hline Exames & Parâmetros & Causas/Significado de valores \\
\hline Ácido úrico & $\begin{array}{l}\mathrm{M}: 3,4-7,0 \mathrm{mg} / \mathrm{dL} \\
\mathrm{F}: 2,4-6,0 \mathrm{mg} / \mathrm{dL}\end{array}$ & $\begin{array}{l}\uparrow \text { Na gota, insuficiência renal, anorexia, leucemia, doença infecciosa } \\
\text { aguda, câncer metastático, eclâmpsia grave, choque, cetose diabética, } \\
\text { acidose metabólica, intoxicação por chumbo, estresse, alcoolismo, } \\
\text { exercício vigoroso, policitemia, psoríase, hiperuricemia assintomática. } \\
\downarrow \text { Com uso de fármacos antigota (ex. alopurinol, probenecida), doença } \\
\text { de Wilson, câncer. }\end{array}$ \\
\hline Albumina & $3,2-4,5 \mathrm{~g} / \mathrm{dL}$ & $\begin{array}{l}\uparrow \text { Na desidratação. } \\
\downarrow \text { Em edema, doença hepática, má absorção, diarreia, queimaduras, } \\
\text { insuficiência renal crônica, desnutrição, estresse, hiper-hidratação, } \\
\text { câncer, gestação, síndrome nefrótica. }\end{array}$ \\
\hline Cálcio (total) & $\begin{array}{l}\text { Adulto: } 8,4-10,2 \mathrm{mg} / \\
\text { dL } \\
\text { Crianças até } 1 \text { ano: } \\
8,5-11,5 \mathrm{mg} / \mathrm{dL}\end{array}$ & $\begin{array}{l}\uparrow: \text { No câncer, hiperparatireoidismo, insuficiência adrenal, } \\
\text { hipertireoidismo, doença óssea de Paget, imobilização prolongada, } \\
\text { ingestão excessiva de vitamina D ou cálcio, uso a longo prazo de } \\
\text { diuréticos tiazídicos, acidose respiratória, insuficiência renal crônica, } \\
\text { doença granulomatosa. } \\
\downarrow \text { : Na hipoalbuminemia, deficiência de vitamina D, uremia, fósforo } \\
\text { elevado, alcalose, diarreia, hipoparatireoidismo, espru, osteomalácia, } \\
\text { má absorção, pancreatite aguda, hipomagnesemia, privação alimentar } \\
\text { prolongada, uso de esteroides. }\end{array}$ \\
\hline Creatinina & $\begin{array}{l}\text { Até } 6 \text { anos: } 0,3- \\
0,7 \mathrm{mg} / \mathrm{dL} \\
\text { De } 7-12 \text { anos: } 0,4- \\
0,8 \mathrm{mg} / \mathrm{dL} \\
\mathrm{M}: 0,8-1,2 \mathrm{mg} / \mathrm{dL} \\
\text { F: } 0,6-1,0 \mathrm{mg} / \mathrm{dL}\end{array}$ & $\begin{array}{l}\uparrow \text { Na insuficiência renal aguda e crônica, dano muscular, } \\
\text { hipertireoidismo, com aumento da massa muscular, privação alimentar } \\
\text { prolongada, acidose diabética, ingestão excessiva de carne, gigantismo, } \\
\text { acromegalia. } \\
\downarrow \text { Na gestação, redução de massa marcular. }\end{array}$ \\
\hline Ferro & $\begin{array}{l}\text { M: } 49-181 \mathrm{~g} / \mathrm{dL} \\
\mathrm{F}: 37-170 \mathrm{~g} / \mathrm{dL}\end{array}$ & $\begin{array}{l}\uparrow \text { Na ingestão excessiva de ferro, anemias hemolíticas, doença hepática, } \\
\text { uso de estrogênio, hemocromatose. } \\
\downarrow \text { Na anemia pode deficiência de cobre, doenças crônicas (ex. lúpus, } \\
\text { artrite reumatoide), infecções, doença hepática, hemorragia, hemólise, } \\
\text { hemoglobinúria, desnutrição, acloridria, cirurgia, infarto do miocárdio, } \\
\text { doenças ou ressecção duodenal e/ou jejunal, uso de antiácidos. }\end{array}$ \\
\hline Globulina & $13-36 \mathrm{mg} / \mathrm{dL}$ & $\begin{array}{l}\uparrow \text { Na desidratação. } \\
\downarrow \text { Em edema, doença hepática, má absorção, diarreia, queimaduras, } \\
\text { insuficiência renal crônica, desnutrição, estresse, hiper-hidratação, } \\
\text { câncer, gestação, síndrome nefrótica. }\end{array}$ \\
\hline Hematócrito & $\begin{array}{l}\text { M: } 40-50 \% \\
\text { F: } 35-45 \%\end{array}$ & $\begin{array}{l}\uparrow \text { Na desidratação, policitemia, choque. } \\
\downarrow \text { Na anemia (Hematócrito }<30 \text { ), perda sanguínea, hemólise, leucemia, } \\
\text { cirrose, hiper-hidratação. }\end{array}$ \\
\hline Hemoglobina & $\begin{array}{l}\text { M: } 13,5-18 \mathrm{~g} / \mathrm{dL} \\
\text { F: } 12-16 \mathrm{~g} / \mathrm{dL}\end{array}$ & $\begin{array}{l}\uparrow \text { Em queimaduras graves, policitemia, insuficiência cardíaca, talassemia, } \\
\text { doença pulmonar obstrutiva crônica, desidratação. } \\
\downarrow \text { Na anemia, hipertireoidismo, cirrose, várias doenças sistêmicas } \\
\text { (Hodgkin, leucemia, lúpus), HIV. }\end{array}$ \\
\hline $\begin{array}{l}\text { Índice PCR/ } \\
\text { Albumina }\end{array}$ & $\begin{array}{l}\text { Sem risco }>0,4 \\
\text { Baixo risco } 0,4-1,2 \\
\text { Médio risco } 1,2-2,0 \\
\text { Alto risco }>2,0\end{array}$ & $\begin{array}{l}\text { Índice classificatório dos graus de risco de complicações do estresse } \\
\text { inflamatório. }\end{array}$ \\
\hline Leucócito & $\begin{array}{l}4.5-11 \times 10^{3} \text { céls/ } \\
\mathrm{mm}^{3}(\mathrm{~mL})\end{array}$ & $\begin{array}{l}\uparrow: \text { Na leucemia, infecção bacteriana, hemorragia, trauma ou injúria } \\
\text { tissular, câncer. } \\
\downarrow \text { : Em algumas infecções virais, quimioterapia, radiação, depressão da } \\
\text { medula óssea, HIV. }\end{array}$ \\
\hline
\end{tabular}


Quadro 2. Exames bioquímicos, parâmetros e interpretações em PVHA.

\begin{tabular}{|c|c|c|}
\hline Exames & Parâmetros & Causas/Significado de valores \\
\hline Linfócito & $\begin{array}{l}20-50 \% \\
1500-5000 \mathrm{~mm}^{3}\end{array}$ & $\begin{array}{l}\uparrow: \text { Em hepatite viral, infecção por citomegalovírus, toxoplasmose, } \\
\text { rubéola, infecção aguda por HIV, leucemia linfocítica crônica e aguda. } \\
\downarrow \text { : Em infecções e enfermidades agudas, doença de Hodgkin, lúpus, } \\
\text { anemia aplástica, insuficiência renal, AIDS, carcinoma terminal. } \\
\text { Desnutrição: } \\
\text { Leve }=1200-1500 \mathrm{~mm}^{3} \\
\text { Moderada }=800-1200 \mathrm{~mm}^{3} \\
\text { Grave }=<800 \mathrm{~mm}^{3}\end{array}$ \\
\hline $\begin{array}{l}\text { Proteínas } \\
\text { totais }\end{array}$ & $6,3-8,1 \mathrm{~g} / \mathrm{dL}$ & $\begin{array}{l}\uparrow: \text { Na desidratação, doenças que elevam os níveis de globulina. } \\
\downarrow: \text { Na deficiência proteica, doença hepática grave, má absorção, diarreia, } \\
\text { queimaduras graves ou infecção, edema, síndrome nefrótica. }\end{array}$ \\
\hline $\begin{array}{l}\text { Relação } \\
\text { Albumina/ } \\
\text { globulina }\end{array}$ & $\begin{array}{l}\text { A/G }<1 \\
\text { A/G }>1\end{array}$ & $\begin{array}{l}\uparrow \text { Melhora do processo inflamatório, início de processo anabólico. } \\
\downarrow \text { Doença renal, anasarca, edema de alça intestinal, processo inflamatório } \\
\text { ativo, fase catabólica. }\end{array}$ \\
\hline TGP & $4-36 \mathrm{U} / \mathrm{L}$ & $\begin{array}{l}\uparrow \text { hepatite, icterícia, cirrose, câncer hepático, infarto do miocárdio, } \\
\text { queimadura severa, trauma, choque, mononucleose, pancreatite, } \\
\text { obesidade. }\end{array}$ \\
\hline TGO & $8-33 \mathrm{U} / \mathrm{L}$ & $\begin{array}{l}\uparrow \text { injúria/ morte celular, infarto miocárdio, cirrose aguda, hepatite, } \\
\text { pancreatite, doença renal, câncer, alcoolismo, hipotireodismo, } \\
\text { queimadura, trauma, distrofia muscular. } \\
\downarrow \text { diabetes não-controlada (acidose), beribéri }\end{array}$ \\
\hline Ureia & $10-45 \mathrm{mg} / \mathrm{dL}$ & $\begin{array}{l}\uparrow \mathrm{Na} \text { insuficiência renal, choque, trauma, desidratação, infecção, } \\
\text { diabetes, gota crônica/catabolismo proteico excessivo, hemorragias } \\
\text { digestivas, infarto do miocárdio, uso de esteroides. } \\
\downarrow \text { Na insuficiência hepática, desnutrição, má absorção, hiper-hidratação, } \\
\text { gestação, diarreia. }\end{array}$ \\
\hline Vitamina B12 & $190-900 \mathrm{ng} / \mathrm{dL}$ & $\begin{array}{l}\uparrow>1100 \mathrm{ng} / \mathrm{dL}: \text { Na doença hepática, algumas leucemias, câncer } \\
\text { (especialmente com metástase hepática), gestação, uso de contraceptivos } \\
\text { orais. } \\
\downarrow<100 \mathrm{ng} / \mathrm{dL}: \mathrm{Na} \text { anemia perniciosa, síndromes de má absorção, } \\
\text { hipotireoidismo primário, redução da mucosa gástrica (ex. gastrectomia } \\
\text { ou câncer gástrico), dieta vegetariana, acloridria. }\end{array}$ \\
\hline Vitamina D & $\begin{array}{l}<10 \mathrm{ng} / \mathrm{dL}: \\
\text { deficiência } \\
<20 \mathrm{ng} / \mathrm{dL}: \\
\text { insuficiência } \\
\geq 30 \mathrm{ng} / \mathrm{dL}: \\
\text { suficiência }\end{array}$ & $\begin{array}{l}\uparrow \text { ingestão excessiva de suplementos, hipercalemia, doença } \\
\text { granulomatosa, sarcoidose, linfoma. } \\
\downarrow \text { HIV/AIDS, desnutrição, osteopenia, doença cardiovascular, obesidade, } \\
\text { insuficiência renal crônica, pele escura. }\end{array}$ \\
\hline
\end{tabular}

$\downarrow$ : Redução; $\uparrow:$ Aumento; TGO: transaminase glutâmico-oxalacética; TGP: transaminase glutâmico-pirúvica; HIV: Human Immunodeficiency Virus; AIDS: Acquired Immunodeficiency Syndrome.

Fontes: European AIDS Clinical Society ${ }^{12}$, Corrêa et al..$^{29}$, Duarte e Borges ${ }^{30}$, Martins et al. ${ }^{31}$.

Quadro 3. Valores de glicose plasmática $(\mathrm{mg} / \mathrm{dL})$ para diagnóstico de diabetes melitus e seus estágios préclínicos.

\begin{tabular}{|l|c|c|}
\hline \multicolumn{1}{|c|}{ Categoria } & Jejum & 2 horas após 75 g de glicose \\
\hline Glicemia normal & $<100$ & $<140$ \\
\hline Tolerância à glicose diminuída & $\geq 100 \mathrm{a}<126$ & $\geq 140 \mathrm{a}<200$ \\
\hline Diabetes Mellitus & $\geq 126$ & $\geq 200$ \\
\hline
\end{tabular}

Fonte: Sociedade Brasileira de Diabetes ${ }^{35}$. 
Quadro 4. Valores referenciais e de alvo terapêutico do perfil lipídico.

\begin{tabular}{|l|c|c|r|}
\hline \multicolumn{1}{|c|}{ Lipídeos } & Com jejum $(\mathbf{m g} / \mathbf{d L})$ & Sem jejum $\mathbf{( m g / d L )}$ & Categoria referencial \\
\hline Colesterol total & $<190$ & $<190$ & Desejável \\
\hline HDL-c & $>40$ & $>40$ & Desejável \\
\hline Triglicerídeos & $<150$ & $<175$ & Desejável \\
\hline \multicolumn{2}{|c|}{ Categoria de risco } & Baixo \\
\hline \multirow{2}{*}{ LDL-c } & $<130$ & $<130$ & Intermediário \\
\cline { 2 - 4 } & $<100$ & $<100$ & Alto \\
\cline { 2 - 4 } & $<70$ & $<70$ & Muito alto \\
\cline { 2 - 4 } & $<50$ & $<50$ & Baixo \\
\hline \multirow{2}{*}{ Não HDL-c } & $<160$ & $<160$ & Intermediário \\
\cline { 2 - 4 } & $<130$ & $<130$ & Alto \\
\cline { 2 - 4 } & $<100$ & $<80$ & Muito alto \\
\cline { 2 - 4 }
\end{tabular}

HDL-c: High-density lipoprotein; LDL-c: Low-density lipoprotein.

Fonte: Sociedade Brasileira e Cardiologia ${ }^{34}$.

tado final um escore ou pontuação que identifica o risco e o estado nutricional de cada indivíduo e são utilizados em pacientes institucionalizados.

A triagem nutricional deve ser realizada em até 72 horas após hospitalização, porém o mais recomendado é que seja realizada até 48 horas e antes da avaliação clínico nutricional, para reconhecer o risco nutricional e possibilitar a instituição de medidas de intervenção que previnam a deterioração do estado nutricional ou promovam sua recuperação e monitorização o mais precocemente ${ }^{39}$. $\mathrm{O}$ instrumento de triagem mais utilizado é o Nutritional Risk Screening - Triagem de Risco Nutricional (NRS-2002) ${ }^{40}$. A NRS-2002, avalia os critérios: perda de peso dos últimos três meses, IMC, ingestão alimentar (apetite e capacidade de se alimentar) e fator de estresse ${ }^{40}$. Em pacientes criticamente enfermos o Nutrition Risk in the Critically Ill (Nutric Score) é o instrumento recomendado ${ }^{41}$. O Nutric Score possibilita identificar o risco de o doente desenvolver efeitos adversos que possam contribuir para o aumento da mortalidade, tais como, instituição de ventilação mecânica, novas infecções, os quais podem ser modificáveis pela terapia nutricional ${ }^{41}$.

A avaliação do estado nutricional deve ser realizada após a detecção do risco nutricional pelos instrumentos de triagem, pois aumenta a sensibilidade e a especificidade dos testes na predição de desfechos clínicos negativos ${ }^{40}$. Assim, a terapia nutricional pode ser direcionada para pacientes efetivamente em risco nutricional ${ }^{40}$. A Avaliação Subjetiva Global (ASG) ${ }^{42}$, Mini Avaliação Nutricional (MAN) ${ }^{43}$ e Avaliação Subjetiva Global Produzida pelo Paciente (ASG-PPP) ${ }^{44}$ são exemplos de instrumentos padronizados para identificar o estado nutricional de pacientes hospitalizados. A ASG, MAN E ASG-PPP são ferramentas aplicadas após a NRS-2002, perante diagnóstico de risco nutricional, para aqueles pacientes que não apresentam risco nutricional não há necessidade.

\section{Considerações finais}

O diagnóstico nutricional de PVHA é muito importante para instituição de tratamento adequado e de prevenção de agravos em todas as etapas do acompanhamento de saúde. Conhecer a situação nutricional das PVHA em tratamento ambulatorial ou hospitalar é a etapa inicial e imprescindível para o sucesso do tratamento dietoterápico a ser instituído. Assim, o diagnóstico nutricional deve ser realizado de forma abrangente, considerando não apenas a situação nutricional atual que os pacientes se encontram, mas acima de tudo conhecer os fatores associados ao desenvolvimento de outras doenças, possibilitando a intervenção precoce e assim, preservar outros aspectos da saúde visando melhorar a qualidade de vida dos pacientes.

Esse trabalho apresentou revisão de protocolos nacionais e internacionais demonstrando a base teórica e conceitual atualizada sobre o diagnóstico nutricional, de forma a contribuir com as equipes de saúde, especialmente os nutricionistas, que atuam no tratamento de pessoas com HIV/ AIDS, bem como no aprimoramento e formação acadêmica nos níveis de graduação e pós-graduação. Com a cronificação da doença e os conheci- 
Quadro 5. Sinais físicos indicativos ou sugestivos de desnutrição.

\begin{tabular}{|c|c|c|c|}
\hline $\begin{array}{c}\text { Área } \\
\text { corporal }\end{array}$ & Aparência normal & Sinais associados com desnutrição & $\begin{array}{c}\text { Doença possível } \\
\text { ou deficiência de } \\
\text { nutriente }\end{array}$ \\
\hline Cabelo & $\begin{array}{l}\text { Firme } \\
\text { Brilhante } \\
\text { Difícil de arrancar }\end{array}$ & $\begin{array}{l}\text { Perda do brilho natural } \\
\text { Seco e feio } \\
\text { Fino e esparso } \\
\text { Seroso e quebradiço } \\
\text { Fino } \\
\text { Despigmentado }\end{array}$ & $\begin{array}{l}\text { Kwashiorkor e, menos } \\
\text { comum, marasmo }\end{array}$ \\
\hline
\end{tabular}


mentos científicos atuais ampliou-se ainda mais a complexidade do diagnóstico e do monitoramento da situação clínica desta população.

Para instituir o plano de cuidado nutricional é necessário pensar sobre a racionalização dos processos de trabalho e gestão do cuidado para que o diagnóstico nutricional completo possa superar barreiras iniciais de implantação. Devido à abrangência do diagnóstico nutricional, bem como complexidade de se instituir novas práticas em serviços de saúde, pode haver dificuldades nas rotinas das equipes e déficit de profissionais para a implantação de um novo protocolo. Assim, destacamos que o mesmo pode ser realizado em etapas e ser viabilizado paulatinamente, de forma a ser racionalizado e incorporado à rotina dos serviços de saúde ${ }^{45-47}$.

Este trabalho pode auxiliar os gestores e equipes de saúde que visam estruturar e/ou aperfeiçoar serviços de referência em tratamento de PVHA, para que o diagnóstico nutricional seja mais valorizado no conjunto das abordagens praticadas. Para que a implementação seja, bemsucedida várias etapas devem ser percorridas, sendo importante a inclusão dos trabalhadores de saúde na sua concepção e estruturação, para que a mudança ocorra efetivamente dentro dos serviços de saúde e beneficie as PVHA.

\section{Colaboradores}

As autoras são responsáveis por todos os aspectos do trabalho, garantindo que as questões relacionadas à precisão ou integridade de qualquer parte do trabalho sejam devidamente investigadas e resolvidas. 


\section{Referências}

1. The Joint United Nations Programme on HIV/AIDS (UNAIDS). How AIDS changed everything: MDG 6: 15 years, 15 lessons of hope from the AIDS response. Geneva: UNAIDS; 2015.

2. Joint United Nations Program on HIV/AIDS (UNAIDS). Fast-track: ending the AIDS epidemic by 2030. Geneva: UNAIDS; 2014.

3. Joint United Nations Program on HIV/AIDS (UNAIDS). Report on the global AIDS epidemic. Geneva: UNAIDS; 2013.

4. Lang S, Boccara F, Mary-Krause M, Cohen A. Epidemiology of coronary heart disease in HIV-infected versus uninfected individuals in developed countries. Arch Cardiovasc Dis 2015; 108(3):206-215.

5. Worm SW, Friis-Møller N, Bruyand M, D’Arminio Monforte A, Rickenbach M, Reiss P, El-Sadr W, Phillips A, Lundgren J, Sabin C, D:A:D study group. High prevalence of the metabolic syndrome in HIV -infected patients: impact of different definitions of the metabolic syndrome. AIDS 2010; 24(3):427-435.

6. Ratien DJ, Mulligan K, Papathakis P, Wanke C. Executive summary-nutritional care of HIV-infected adolescents and adults, including pregnant and lactating women: what do we know, what can we do, and where do we go from here? Am J Clin Nutr 2011; 94(Supl.):1667S-1676S.

7. Soares GB, Garbin CAS, Rovida TAS, Garbin AJÍ Quality of life of people living with HIV/AIDS treated by the specialized service in Vitória-ES, Brazil. Cien Saude Colet 2015; 20(4):1075-1084.

8. Associação Brasileira de Nutrição (ASBRAN). Manual orientativo: sistematização do cuidado de nutrição. São Paulo: ASBRAN; 2014.

9. Brasil. Ministério da Saúde (MS). Protocolo clínico e diretrizes terapêuticas para manejo da infecção pelo HIV em adultos. Brasília: MS; 2018.

10. Fields-Gardner C, Campa A, American Dietetics Association. Position of the American Dietetic Association: nutrition intervention and human immunodeficiency virus infection. J Am Diet Assoc 2010; 110(7):1105-1119.

11. U.S. Department of Health and Human Services. Health Resources and Services Administration. Guide for HIV/AIDS Clinical Care - 2014. Rockville: U.S. Department of Health and Human Services; 2014.

12. European Aids Clinical Society (EACS). Guidelines: Version 9.1. Zurich: EACS; 2018

13. Ockenga J, Grimble R, Jonkers-Schuitema C, Macallan D, Melchior JC, Sauerwein HP, Schwenk A, Dgem (German Society for Nutritional Medicine), Süttmann U, ESPEN (European Society for Parenteral and Enteral Nutrition). ESPEN Guidelines on Enteral Nutrition: Wasting in HIV and other chronic infectious diseases. Clin Nut 2006; 25(2):319-329.

14. Wanke C, Polsky B, Kotler D. Guidelines for using body composition measurement in patients with human immunodeficiency virus infection. AIDS Patient Care STDS 2002; 16(8):375-388.

15. World Health Organization (WHO). Guidelines for the clinical management of HIV infection in adults and adolescents. National AIDS-STD Control Programme. Department of Health. Geneva: WHO; 2007.
16. World Health Organization (WHO). Nutrient requirements for people living with HIV/Aids. Reports of a technical consultation. Geneva: WHO; 2003.

17. Falco MO, Silveira EA. Nutrition therapy for HAARTnaïve HIV-infected patients. In: Watson RR, editor Health of HIV infected people: food, nutrition, lifestyle without antiretroviral drugs. San Diego: Elsevier; 2015. p. 95-111.

18. Silveira EA, Falco MO. Nutrition Therapy for Adults with HIV on ART. In: Watson RR, editor. Health of HIV infected people: food, nutrition, lifestyle with antiretroviral drugs. San Diego: Elsevier; 2015. p. 307-337.

19. Brasil. Ministério da Saúde (MS). Manual clínico de alimentação e nutrição na assistência a adultos infectados pelo HIV. Brasília: MS; 2006.

20. Brasil. Ministério da Saúde (MS). Secretaria de Vigilância em Saúde. Departamento de DST, Aids e hepatites virais. Protocolo clínico e diretrizes terapêuticas para manejo da infecção pelo HIV em adultos. Brasília: MS; 2013.

21. Alves MD, Brites C, Sprinz E. HIV-associated lipodystrophy: a review from a Brazilian perspective. Ther Clin Risk Manag 2014; 17(10):559-566.

22. Chen D, Misra A, Garg A.Clinical review 153: lipodystrophy in human immunodeficiency virus-infected patients. J Clin Endocrinol Metab 2002; 87(11):4845-4856

23. Carr A, Cooper DA. A. Adverse effects of antiretroviral therapy. Lancet 2000; 356(9239):1423-1430.

24. Finkelstein JL, Gala P, Rochford R, Glesby MJ, Mehta S. HIV/AIDS and lipodystrophy: implications for clinical management in resource-limited settings. J Int AIDS Soc 2015; 18(1):19033.

25. Mutimura E, Crowther NJ, Cade TW, Yarasheski KE, Stewart A. Exercise training reduces central adiposity and improves metabolic indices in HAART-treated HIV-positive subjects in Rwanda: a randomized controlled trial. AIDS Res Hum Retroviruses 2008; 24(1):15-23.

26. World Health Organization (WHO). Obesity: preventing and managing the global epidemic. Report of a WHO Consultation. Geneva: WHO; 2000.

27. Heyward VH. ASEP Methods recommendation: body composition assessment. J Exercise Physiol 2001, 4(4):1-12.

28. Guimarães NS, Faustob MA, Kakehasia AM, Navarroc AM, Tupinambása U. Can anthropometry measure the body fat of people living with HIV/AIDS? A systematic review. Rev Esp Nutr Hum Diet 2017; 21(2):101111.

29. Corrêa CR, Angeleli AYO, Camargo NR, Barbosa L, Burin RC. Comparação entre a relação PCR/albumina e o índice prognóstico inflamatório nutricional (IPIN). Jornal Bras Patol Med Laboratorial 2002; 38(3):183-190

30. Duarte ACG, Borges VLS. Semiologia nutricional. In: Duarte ACG, organizador. Avaliação nutricional: aspectos clínicos e laboratoriais. São Paulo: Atheneu; 2007. p. 21-28.

31. Martins C, Moreira SM, Pierosan SR. Interações droga -nutrientes. São Paulo: Varela; 2001.

32. Nixon DE, Landay AL. Biomarkers of immune dysfunction in HIV. Curr Opin HIV AIDS 2010; 5(6):498503. 
33. Hileman CO, Funderburg NT. Inflammation, Immune Activation and Antiretroviral Therapy in HIV. Curr HIV/AIDS Rep 2017; 14(3):93-100.

34. Sociedade Brasileira de Cardiologia (SBC). Atualização da diretriz brasileira de dislipidemias e prevenção da aterosclerose - 2017. Arq Bras Cardiol 2017; 109(2):1-76.

35. Sociedade Brasileira de Diabetes (SBD). Diretrizes da sociedade brasileira de diabetes. São Paulo: SBD; 2016.

36. Tiozzo E, Konefal J, Adwan S, Martinez LA, Villabona J, Lopez J, Cutrono S, Mehdi SM, Rodriguez A, Woolger JM, Lewis JE. A cross-sectional assessment of metabolic syndrome in HIV-infected people of low socio-economic status receiving antiretroviral therapy. Diabetol Metab Syndr 2015; 7(7):1-15.

37. Sociedade Brasileira de Cardiologia (SBC). $7^{a}$ diretriz brasileira de hipertensão arterial. Arq Bras Cardiol 2016; 107(3):1-19.

38. Vannucchi H, Unamuno MRDL, Marchini JS. Avaliação do estado nutricional. Medicina (Ribeirão Preto) 1996; 29(1):5-18.

39. Dias MCG, van Aanholt DPJ, Catalani LA, Rey JSF, Gonzales MC, Coppini L, Franco Filho JW, Paes-Barbosa MR, Horie L, Abrahão V, Martins C. Sociedade Brasileira de Nutrição Parenteral e Enteral (SBNPE). Associação Brasileira de Nutrologia. Projeto diretrizes: triagem e avaliação do estado nutricional. São Paulo; 2011.

40. Raslan M, Gonzalez MC, Dias MCG, Paes-Barbosa FC, Cecconello I, WaitzBerg DL. Aplicabilidade dos métodos de triagem nutricional no paciente hospitalizado. Rev Nutrição 2008; 21(5):553-561.

41. Heyland DK, Dhaliwal R, Jiang X, Day AG. Identifying critically ill patients who benefit the most from nutrition therapy: the development and initial validation of a novel risk assessment tool. Crit Care 2011; 15(R268):1-11.

42. Detsky AS, McLaughlin JR, Baker JP, Johnston N, Whittaker S, Mendelson RA, Jeejeebhoy KN. What is subjective global assessment of nutritional status? JPEN. J Parenter Enteral Nutr 1987; 11(1):8-13.
43. Machado RS, Coelho MA, Veras RP. Validity of the portuguese version of the mini nutritional assessment in brazilian elderly. BMC Geriatrics 2015; 22(15):132.

44. Duarte BCJA, Dias PC. Cross-cultural adaptation of the Portuguese version of the patient-generated subjective global assessment. Nutr Hosp 2012; 27(2):583589.

45. Azevedo CS, Sá MC, Cunha M, Matta GC, Miranda L, Grabois V. Racionalização e Construção de Sentido na Gestão do Cuidado: uma experiência de mudança em um hospital do SUS. Cien Saude Colet 2017; 22(6):1991-2002.

46. Melo EA, Maksud I, Agostini R. Cuidado, HIV/Aids e atenção primária no Brasil: desafio para a atenção no Sistema Único de Saúde? Rev Panam Salud Publica 2018; 42:e151.

47. Scherer MDA, Conill EM, Jean R, Taleb A, Gelbcke FL, Pires DEP, Joazeiro EMG. Desafios para o trabalho em saúde: um estudo comparado de Hospitais Universitários na Argélia, Brasil e França. Cien Saude Colet 2018; 23(7):2265-2276.

Artigo apresentado em 21/02/2018

Aprovado em 15/04/2019

Versão final apresentada em 17/04/2019 